\title{
Mutation signatures implicate aristolochic acid in bladder cancer development
}

\author{
Song Ling Poon ${ }^{1,2 \dagger}$, Mi Ni Huang ${ }^{2,3+}$, Yang Choo ${ }^{2,3}$, John R McPherson ${ }^{2,3}$, Willie Yu ${ }^{1,2,4}$, Hong Lee Heng ${ }^{1,2}$, \\ Anna Gan ${ }^{1,2}$, Swe Swe Myint ${ }^{1,2}$, Ee Yan Siew ${ }^{1,2}$, Lian Dee Ler ${ }^{1,2,4}$, Lay Guat Ng ${ }^{5}$, Wen-Hui Weng ${ }^{6}$, \\ Cheng-Keng Chuang ${ }^{7}$, John SP Yuen ${ }^{5}$, See-Tong Pang ${ }^{7^{*}}$, Patrick Tan ${ }^{2,8,9,10^{*}}$, Bin Tean Teh ${ }^{1,2,9^{*}}$ \\ and Steven G Rozen ${ }^{2,3^{*}}$
}

\begin{abstract}
Background: Aristolochic acid (AA) is a natural compound found in many plants of the Aristolochia genus, and these plants are widely used in traditional medicines for numerous conditions and for weight loss. Previous work has connected AA-mutagenesis to upper-tract urothelial cell carcinomas and hepatocellular carcinomas. We hypothesize that AA may also contribute to bladder cancer.

Methods: Here, we investigated the involvement of AA-mutagenesis in bladder cancer by sequencing bladder tumor genomes from two patients with known exposure to AA. After detecting strong mutational signatures of AA exposure in these tumors, we exome-sequenced and analyzed an additional 11 bladder tumors and analyzed publicly available somatic mutation data from a further 336 bladder tumors.

Results: The somatic mutations in the bladder tumors from the two patients with known AA exposure showed overwhelming AA signatures. We also detected evidence of AA exposure in 1 out of 11 bladder tumors from Singapore and in 3 out of 99 bladder tumors from China. In addition, 1 out of 194 bladder tumors from North America showed a pattern of mutations that might have resulted from exposure to an unknown mutagen with a heretofore undescribed pattern of $A>T$ mutations. Besides the signature of AA exposure, the bladder tumors also showed the CPG > TPG and activated-APOBEC signatures, which have been previously reported in bladder cancer.

Conclusions: This study demonstrates the utility of inferring mutagenic exposures from somatic mutation spectra. Moreover, AA exposure in bladder cancer appears to be more pervasive in the East, where traditional herbal medicine is more widely used. More broadly, our results suggest that AA exposure is more extensive than previously thought both in terms of populations at risk and in terms of types of cancers involved. This appears to be an important public health issue that should be addressed by further investigation and by primary prevention through regulation and education. In addition to opportunities for primary prevention, knowledge of AA exposure would provide opportunities for secondary prevention in the form of intensified screening of patients with known or suspected AA exposure.
\end{abstract}

\footnotetext{
* Correspondence: jacobpang@adm.cgmh.org.tw; gmstanp@duke-nus.edu.sg; teh.bin.tean@singhealth.com.sg; steve.rozen@duke-nus.edu.sg

${ }^{\dagger}$ Equal contributors

${ }^{7}$ Division of Urology, Department of Surgery, Chang Gung Memorial Hospital, Linkou, School of Medicine, Chang Gung University, 5, Fusing Street, Gueishan Township, Taoyuan County 333, Taiwan

${ }^{2}$ Program in Cancer and Stem Cell Biology, Duke-NUS Graduate Medical School, 8 College Road, Singapore 169857, Singapore

${ }^{1}$ Laboratory of Cancer Epigenome, Division of Medical Sciences, National Cancer Centre Singapore, 11 Hospital Drive, Singapore 169610, Singapore Full list of author information is available at the end of the article
} 


\section{Background}

Bladder cancer is a major cause of morbidity and mortality worldwide, causing an estimated 150,000 deaths per year [1]. In developed countries, approximately $90 \%$ of bladder cancers are urothelial cancers, that is, cancers of the transitional cells that line the bladder. These cancers can be subclassified as muscle-invasive or nonmuscle-invasive. Exposure to environmental carcinogens has long been known to cause bladder cancers. The most prominent of these exposures is inhaled tobacco smoke [2,3]. Additional environmental causes include arsenic [4,5] and certain occupational exposures, such as aromatic amines [6-9]. Chlorinated drinking water is also a possible contributing exposure [10].

An emerging field known as molecular epidemiology augments classical epidemiological approaches with exposure-associated molecular biomarkers to better substantiate causal links. This has significant implications in both clinical oncology and public health. Indeed, inexpensive next-generation sequencing provides a new molecular epidemiological tool for inferring environmental exposures and endogenous mutational processes that occurred prior to and during oncogenesis [11-17]. Somatic mutations can be identified by finding genetic variants in tumors and subtracting from them the variants found in non-malignant tissues from the same individual. Different environmental exposures and endogenous mutagenic processes often have characteristic somatic mutation signatures in terms of single nucleotide changes. These signatures often include preferences for particular bases $5^{\prime}$ and $3^{\prime}$ of the location of the mutation, for example, a preference for $\mathrm{G}$ (guanosine) $3^{\prime}$ of $\mathrm{C}>\mathrm{T} \mathrm{mu}-$ tations, reflecting mutations caused by deamination of 5-methyl cytosine in CpG > TpG contexts. Often tumors show overlays of multiple mutational exposures and processes, which can be deconvolved by approaches based on non-negative matrix factorization (NMF) or software that uses related methods $[16,18,19]$.

Recently, the mutation spectra of bladder tumors from North America and Denmark have been analyzed, and three major signatures were detected [16,17,20-22]. One of these is thought to result from activated APOBEC genes. The second signature corresponds to the aforementioned deamination of 5-methyl cytosines in CpGs. The final mutation signature, Signature 5 [16], is of uncertain origin but partially correlates with tobacco smoking [16].

We and others recently showed that aristolochic acid (AA), which is present in many plants in the genus Aristolochia that are used in traditional herbal medicine, induces highly distinctive mutation signatures in the genomes of upper urinary tract urothelial cell carcinomas (UTUC) [14,23-28]. We also found probable AA mutation signatures in hepatocellular carcinomas (HCCs) from southern China, where herbal medicine is widely used, suggesting that AA is a contributory factor in HCC development in this region [14]. Subsequently, AA mutation signatures were detected in renal cell carcinomas (RCCs) from Romania and Croatia [29,30]. The AA signatures were found both in patients from areas where AA exposure was known to be prevalent, but also, importantly, in patients from other areas. These findings in HCCs and RCCs exemplified the utility of mutation signatures for the detection of previously unexpected carcinogenic exposures.

In the study reported here, we investigated whether bladder cancer might also be caused by AA exposure. There is anecdotal evidence that patients with AAinduced kidney failure may have elevated risk of bladder cancer [31-33], and AA-DNA adducts occur in the bladders of AA-fed mice [34,35]. To our knowledge, however, there have been no population-based epidemiological or molecular epidemiological investigations of possible AA exposure in the development of bladder tumors. In the current study, we scrutinized the somatic mutation spectra of 349 bladder cancer exomes to determine if they reveal evidence of AA exposure, starting with tumors from patients with known exposure and then extending the study to tumors from patients with unknown exposure.

\section{Methods}

\section{Clinical samples and information}

Tissue samples and clinical information on two patients with bladder cancer and known AA exposure (130T and 136T) were obtained from the Chang Gung Memorial Hospital in Taiwan. Eleven additional bladder cancers were obtained from Singapore General Hospital in Singapore. Written informed consent was obtained from each subject, the research protocol was approved by the Human Research Ethics Committee of the Chang Gung Memorial Hospital and SingHealth Institutional Review Board, and the research conformed to the Helsinki Declaration. See Table S1 in Additional file 1 for details of the patients treated in Taiwan and Singapore. The somatic mutation data from the 99 bladder cancers treated in China was reported previously [36]. The somatic mutation data from 237 urothelial bladder tumors from The Cancer Genome Atlas (TCGA) [37] were downloaded from TCGA data portal [38] on 8 May 2014. For 130 out of these 237 tumors, somatic data were published previously [39]. Out of the 237 tumors represented in TCGA data, 194 were from patients treated in North America. The treatment locations of the other 43 patients are not clear, as the samples came from biobank organizations. The ethnicities of the 237 TCGA patients were: 'white', 172; 'Asian', 26; 'black or African American', 12; 'unknown', 27. We also analyzed 
previously reported data on 11 HCCs [40], 24 UTUCs and two AA-exposed cell lines [14]. The total number of tumors analyzed was 386 .

\section{Sequencing and identification of somatic mutations}

The genomes of the two AA-associated bladder cancers from Taiwan, the 11 bladder cancers from Singapore, and the respective matched nonmalignant tissue samples were sequenced on an Illumina HiSeq 2000 as pairedend 76-bp reads. Read pairs were aligned to the reference human genome (hg19) using Burrows-Wheeler Aligner [41]. Somatic single nucleotide mutations were identified according to their presence in the tumor genome and absence from the corresponding normal genome using a discovery pipeline based on the Genome Analyzer Toolkit. The sequencing reads for the tumors that were sequenced for this study (Table S2 in Additional file 1) have been deposited at the European Genome-phenome Archive [42] under accession EGAS00001000975.

\section{Identifying mutation signatures with EMu and non-negative matrix factorization}

EMu version 1.5.2 [19] (downloaded from [43]) was used to infer the number of elementary mutational processes and their signatures from the catalogs of somatic mutations in bladder cancer [36,39], UTUC [14], HCC [40], and AA-treated cell line genomes [14]. We ran EMu with default parameters and an 'opportunity file' based on the human exome. We independently confirmed the $\mathrm{EMu}$ analysis with the NMF implementation used previously [16] (Figures S8 to 10 in Additional file 1). We ran NMF with the arguments iterationsPerCore $=10$, minNumberOfSignature $=2$, and $\operatorname{maxNumberOfSignature~}=8$.

\section{Statistical and computational analysis}

Statistical analysis was carried out in $\mathrm{R}$ [44]. We used the binom.test function to test for an excess of $\mathrm{A}: \mathrm{T}>\mathrm{T}: \mathrm{A}$ mutations and to test for an excess of $\mathrm{A}>\mathrm{T}$ mutations on the non-transcribed strand (that is, for strand bias). False discovery rates (FDRs) were calculated using the p.adjust function with the fdr method. We used the cosine function in the R package lsa [45] to calculate cosine similarity.

\section{Results}

\section{Aristolochic acid induces its distinctive mutation} signature in bladder cancer

As an initial investigation into whether AA contributes to somatic mutations in bladder cancer, we performed whole-genome sequencing on two tumors from patients with histories of consuming AA-containing herbal remedies. Neither patient had end-stage renal disease (a common consequence of AA-induced nephrotoxicity) nor a history of UTUC or HCC at their first diagnosis of bladder cancer. We focused on the analysis of the exonic portions of these genomes, which harbored 2,054 somatic single nucleotide substitutions - a total of 1,721 different genes were affected by non-silent mutations. These AA-associated bladder cancers had mutation spectra that were similar to those of previously reported AAassociated UTUCs [14] (Figure 1B). The bladder tumors also showed a predominance of $\mathrm{A}>\mathrm{T}$ transversions that was not seen in previous pan-cancer analyses $[17,18]$ but that is characteristic of AA mutagenesis $[16,17]$. This predominance of $\mathrm{A}>\mathrm{T}$ mutations was coupled with a preference for CAG and TAG contexts (that is, CAG > CTG, TAG > TTG; Figure 1A,B), and the overall pattern of A:T > T:A mutations had cosine similarities ranging from 0.977 to 0.992 . In addition, both bladder tumors had many fewer $\mathrm{A}>\mathrm{T}$ mutations on the transcribed compared with the non-transcribed strands: over $70 \%$ of the $\mathrm{A}>\mathrm{T}$ mutations were on the non-transcribed strand (Table 1). Figure $1 \mathrm{C}$ shows the number of mutations on the transcribed and nontranscribed strands of the two bladder tumors and the associated $P$-values for strand bias. For comparison, Figure 1D shows that the strand bias and $P$-values for two AA-UTUCs are similar, with $>75 \%$ of the A $>$ T mutations on the non-transcribed strand (Table 1).

\section{Aristolochic acid exposure in bladder cancers from other populations}

Having established that AA can contribute to somatic mutations in bladder cancer, we next examined the somatic mutation catalogs from a larger set of 347 bladder cancers for evidence of AA-induced mutations. This set consisted of 11 tumors from Singapore that we sequenced for this study, as well as 336 publicly available whole-exome or whole-genome sequenced tumors. Of these, 99 were non-muscle-invasive tumors from patients treated in China [36], and the remaining 237 were muscle-invasive tumors from patients treated either in North America (194 patients) or at unknown locations (43 patients) [39]. Examination of the somatic mutation spectra of these tumors revealed some with the characteristics of AA exposure in the form of elevated proportions of $\mathrm{A}>\mathrm{T}$ mutations with a marked bias against mutations on the transcribed strand (Figure 2A-E; Figures S2 to S7 in Additional file 1).

To systematically explore this observation, we used $\mathrm{EMu}$ [19] to extract mutation signatures from the exomes of all 349 cases (including the two from Taiwan), plus exomes from previously published AA-exposed and non-exposed UTUCs, HCCs, and cell lines (a total of 37 additional exomes). EMu distinguished three mutation signatures in these tumors (Figure 3A). Each signature was characterized by a different pattern of 


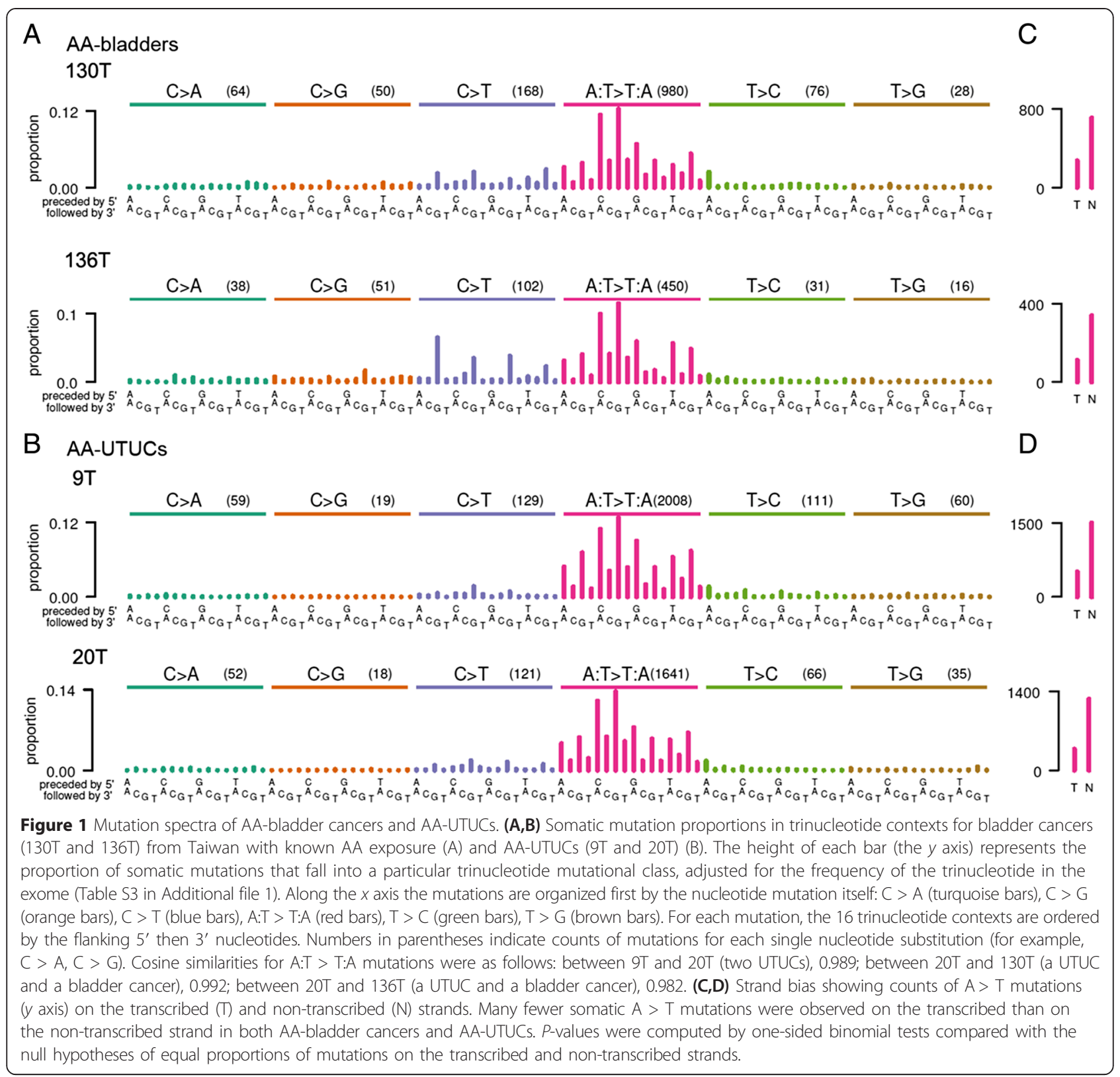

the 96 potential mutations in a trinucleotide context, and each signature contributed to a different proportion of the mutations in each of the tumors (Figure 3B). Notably, EMu discerned a signature almost identical to the mutation spectra observed in UTUCs strongly mutagenized by $\mathrm{AA}$, as can be seen by comparing the EMu AA signature in Figure $3 \mathrm{~A}$ with the spectra in Figure 1B (cosine similarities for A:T > T:A mutations >0.987). To our knowledge, this signature has not previously been reported in bladder cancers $[16,17,20]$. In addition to the AA signature, EMu discerned the previously reported signatures of $\mathrm{CpG}>\mathrm{TpG}$ mutations and of activated APOBECs $(\mathrm{C}>\mathrm{T}$ and $\mathrm{C}>\mathrm{G}$ mutations in
TCW trinucleotide contexts, where $\mathrm{W}$ denotes $\mathrm{A}$ or $\mathrm{T}$; Figure 3A) $[16,17,20]$. To further confirm the EMu analysis, we also analyzed the same set of tumors with NMF $[16,17]$ and found nearly identical signatures (cosine similarity 0.9988 between the EMu and NMF AA signatures) and similar estimates of the AA signature's contributions for these tumors (Figures S8 to S10 in Additional file 1).

As part of their analyses, EMu and NMF estimate the contribution of each mutation signature to the somatic mutations of each tumor (Table 1). However, neither EMu nor NMF provides a statistical hypothesis test of the presence or absence of a particular signature in a 
Table 1 Tumors with high proportions of A:T > T:A mutations

\begin{tabular}{|c|c|c|c|c|c|c|c|c|c|c|c|c|}
\hline \multirow[b]{2}{*}{ Sample } & \multirow[b]{2}{*}{ Cancer type } & \multirow{2}{*}{$\begin{array}{l}\text { Mutations attributed } \\
\text { to } A A \\
\text { Proportion }\end{array}$} & \multicolumn{2}{|c|}{ Mutation count } & \multicolumn{2}{|c|}{ A:T > T:A proportion } & \multicolumn{2}{|l|}{ A $>$ T Count } & \multirow{2}{*}{$\begin{array}{l}\text { Non-transcribed/ } \\
(\text { all } A>T)\end{array}$} & \multicolumn{2}{|c|}{ Strand bias } & \multirow{2}{*}{$\begin{array}{l}\text { A:T > T:A cosine } \\
\text { similarity with } \\
\text { AA signature }\end{array}$} \\
\hline & & & Total & $A: T>T: A$ & $P$ & FDR & Non-transcribed & Transcribed & & $P$ & FDR & \\
\hline 9T & UTUC & 0.97 & 2386 & 2008 & 0 & 0 & 1505 & 503 & 0.75 & $8 \mathrm{E}-116$ & $3 \mathrm{E}-113$ & 0.987 \\
\hline $20 \mathrm{~T}$ & UTUC & 0.96 & 1933 & 1641 & 0 & 0 & 1265 & 376 & 0.77 & $1 \mathrm{E}-112$ & $2 \mathrm{E}-110$ & 0.995 \\
\hline K100T & UTUC & 0.97 & 2342 & 1982 & 0 & 0 & 1435 & 547 & 0.72 & $9 \mathrm{E}-92$ & $1 \mathrm{E}-89$ & 0.992 \\
\hline $6 \mathrm{~T}$ & UTUC & 0.95 & 1552 & 1302 & 0 & 0 & 994 & 308 & 0.76 & $1 E-84$ & $9 \mathrm{E}-83$ & 0.995 \\
\hline K80T & UTUC & 0.98 & 1713 & 1480 & 0 & 0 & 1100 & 380 & 0.74 & $1 \mathrm{E}-81$ & $1 \mathrm{E}-79$ & 0.988 \\
\hline $13 \mathrm{~T}$ & UTUC & 0.99 & 1409 & 1222 & 0 & 0 & 924 & 298 & 0.76 & $3 \mathrm{E}-75$ & $2 E-73$ & 0.994 \\
\hline К79Т & UTUC & 0.99 & 1573 & 1374 & 0 & 0 & 1015 & 359 & 0.74 & $5 E-73$ & $3 \mathrm{E}-71$ & 0.992 \\
\hline 130T & Bladder & 0.82 & 1366 & 980 & 0 & 0 & 708 & 272 & 0.72 & $1 E-45$ & $5 E-44$ & 0.992 \\
\hline HK41T & $\mathrm{HCC}$ & 0.95 & 781 & 630 & 0 & 0 & 441 & 189 & 0.70 & $2 E-24$ & $7 \mathrm{E}-23$ & 0.988 \\
\hline $3 \mathrm{~T}$ & UTUC & 0.91 & 666 & 524 & 0 & 0 & 369 & 155 & 0.70 & $2 \mathrm{E}-21$ & $6 \mathrm{E}-20$ & 0.959 \\
\hline $136 \mathrm{~T}$ & Bladder & 0.73 & 688 & 450 & $1 \mathrm{E}-229$ & $4 \mathrm{E}-228$ & 339 & 111 & 0.75 & $3 E-28$ & $1 \mathrm{E}-26$ & 0.987 \\
\hline 10T & UTUC & 0.73 & 545 & 351 & $3 \mathrm{E}-176$ & $9 \mathrm{E}-175$ & 263 & 88 & 0.75 & $1 \mathrm{E}-21$ & $4 \mathrm{E}-20$ & 0.972 \\
\hline HK2B_8d2 & AA-treated cell line & 0.73 & 413 & 264 & $7 E-132$ & $2 \mathrm{E}-130$ & 178 & 86 & 0.67 & $8 \mathrm{E}-09$ & $2 \mathrm{E}-07$ & 0.972 \\
\hline GZ75T & $\mathrm{HCC}$ & 0.71 & 404 & 250 & $4 \mathrm{E}-120$ & $1 \mathrm{E}-118$ & 167 & 83 & 0.67 & $6 \mathrm{E}-08$ & $2 \mathrm{E}-06$ & 0.975 \\
\hline HK2_AA & AA-treated cell line & 0.66 & 261 & 152 & $2 \mathrm{E}-68$ & $4 \mathrm{E}-67$ & 107 & 45 & 0.70 & $3 \mathrm{E}-07$ & $6 \mathrm{E}-06$ & 0.905 \\
\hline 33324197Т & Bladder & 0.42 & 182 & 67 & $5 E-17$ & $1 \mathrm{E}-15$ & 46 & 21 & 0.69 & $2 \mathrm{E}-03$ & $3 \mathrm{E}-02$ & 0.942 \\
\hline B23 & Bladder & 0.36 & 241 & 76 & $9 \mathrm{E}-15$ & $2 \mathrm{E}-13$ & 56 & 20 & 0.74 & $2 \mathrm{E}-05$ & $4 \mathrm{E}-04$ & 0.949 \\
\hline HK174T & $\mathrm{HCC}$ & 0.48 & 109 & 46 & $1 \mathrm{E}-14$ & $2 \mathrm{E}-13$ & 24 & 22 & 0.52 & 0.44 & 0.85 & 0.886 \\
\hline HK65Т & $\mathrm{HCC}$ & 0.47 & 119 & 48 & $2 \mathrm{E}-14$ & $5 \mathrm{E}-13$ & 41 & 7 & 0.85 & $3 \mathrm{E}-07$ & 7E-06 & 0.889 \\
\hline B77 & Bladder & 0.45 & 106 & 42 & $2 \mathrm{E}-12$ & $4 \mathrm{E}-11$ & 38 & 4 & 0.90 & $3 \mathrm{E}-08$ & $8 \mathrm{E}-07$ & 0.927 \\
\hline HK81T & $\mathrm{HCC}$ & 0.45 & 101 & 39 & $3 \mathrm{E}-11$ & $5 E-10$ & 26 & 13 & 0.67 & $3 \mathrm{E}-02$ & 0.26 & 0.893 \\
\hline GZ119T & $\mathrm{HCC}$ & 0.46 & 59 & 25 & $1 \mathrm{E}-08$ & $2 \mathrm{E}-07$ & 17 & 8 & 0.68 & $5 \mathrm{E}-02$ & 0.40 & 0.736 \\
\hline B89-12 & Bladder & 0.3 & 238 & 62 & $1 \mathrm{E}-08$ & $2 \mathrm{E}-07$ & 48 & 14 & 0.77 & $9 \mathrm{E}-06$ & $2 \mathrm{E}-04$ & 0.950 \\
\hline HK267T & $\mathrm{HCC}$ & 0.43 & 61 & 23 & $5 \mathrm{E}-07$ & $8 \mathrm{E}-06$ & 18 & 5 & 0.78 & $5 \mathrm{E}-03$ & 7E-02 & 0.833 \\
\hline HK75T & $\mathrm{HCC}$ & 0.41 & 67 & 24 & $9 \mathrm{E}-07$ & $1 \mathrm{E}-05$ & 12 & 12 & 0.50 & 0.58 & 1 & 0.871 \\
\hline $21 \mathrm{~T}$ & UTUC & 0.34 & 76 & 24 & $1 \mathrm{E}-05$ & $2 \mathrm{E}-04$ & 17 & 7 & 0.71 & $3 \mathrm{E}-02$ & 0.27 & 0.860 \\
\hline $\begin{array}{l}\text { TCGA-K4-A6FZ-01A- } \\
\text { 11D-A31L-08 }\end{array}$ & Bladder & 0.18 & 461 & 83 & $4 \mathrm{E}-04$ & $6 \mathrm{E}-03$ & 52 & 31 & 0.63 & $1 \mathrm{E}-02$ & 0.15 & 0.525 \\
\hline $1 \mathrm{~T}$ & UTUC & 0.27 & 70 & 18 & $2 \mathrm{E}-03$ & $3 \mathrm{E}-02$ & 17 & 1 & 0.94 & $7 \mathrm{E}-05$ & $1 \mathrm{E}-03$ & 0.777 \\
\hline
\end{tabular}




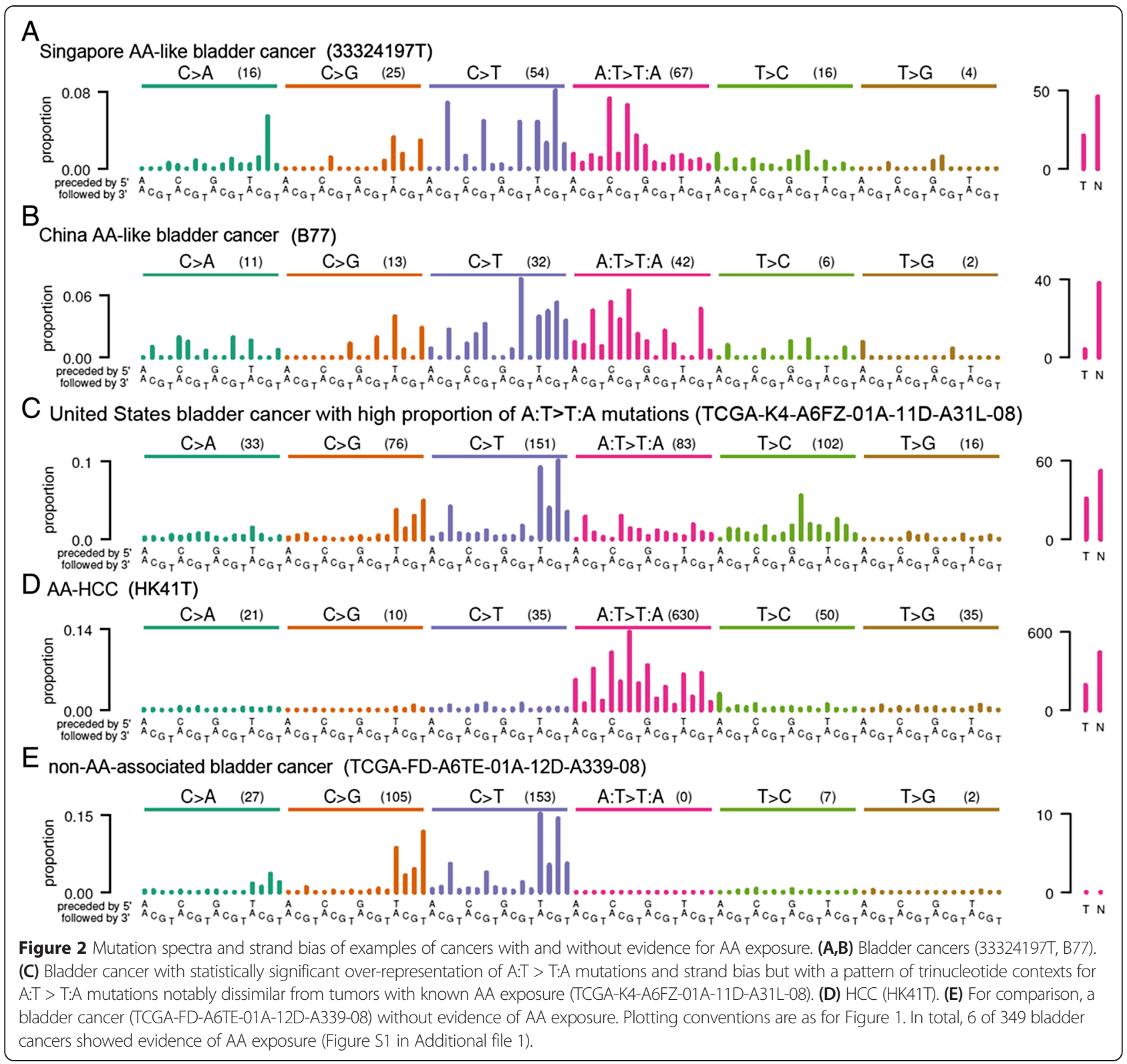

given tumor. Indeed, both EMu and NMF often attribute at least a small number of mutations to every available signature, which for many exposures does not correspond to a biologically plausible level of exposure. Therefore, we developed statistical tests for likely AA exposure based on two characteristics of AA mutagenesis. One was the high proportion of A:T > T:A mutations (Figure 3A), which are rare among other known mutation signatures [16,17]. The other characteristic was the bias against the presence of $\mathrm{A}>\mathrm{T}$ mutations on the transcribed (antisense) strand, which is presumed to result from transcription-coupled repair of AA-derived adenine adducts on this strand. To statistically test for AA exposure, we made use of the high proportion of
A:T > T:A mutations caused by AA and the fact that, among previously reported signatures in bladder cancer, Signature 5 has the highest proportion of A:T > T:A mutations (Figure S7 in Additional file 1; Figure S31 in reference [16]). Specifically, we adopted the null hypothesis that the A:T > T:A mutations in a given tumor were derived only from Signature 5 and calculated as a $P$-value the probability that Signature 5 alone, with no contribution from AA mutagenesis, was responsible for the observed proportion of A:T > T:A mutations. The proportion of A:T > T:A mutations in Signature 5 is $12.5 \%$ [16]. This null hypothesis was conservative in that the other signatures previously observed in bladder tumors have far lower proportions of A:T > T:A mutations than 

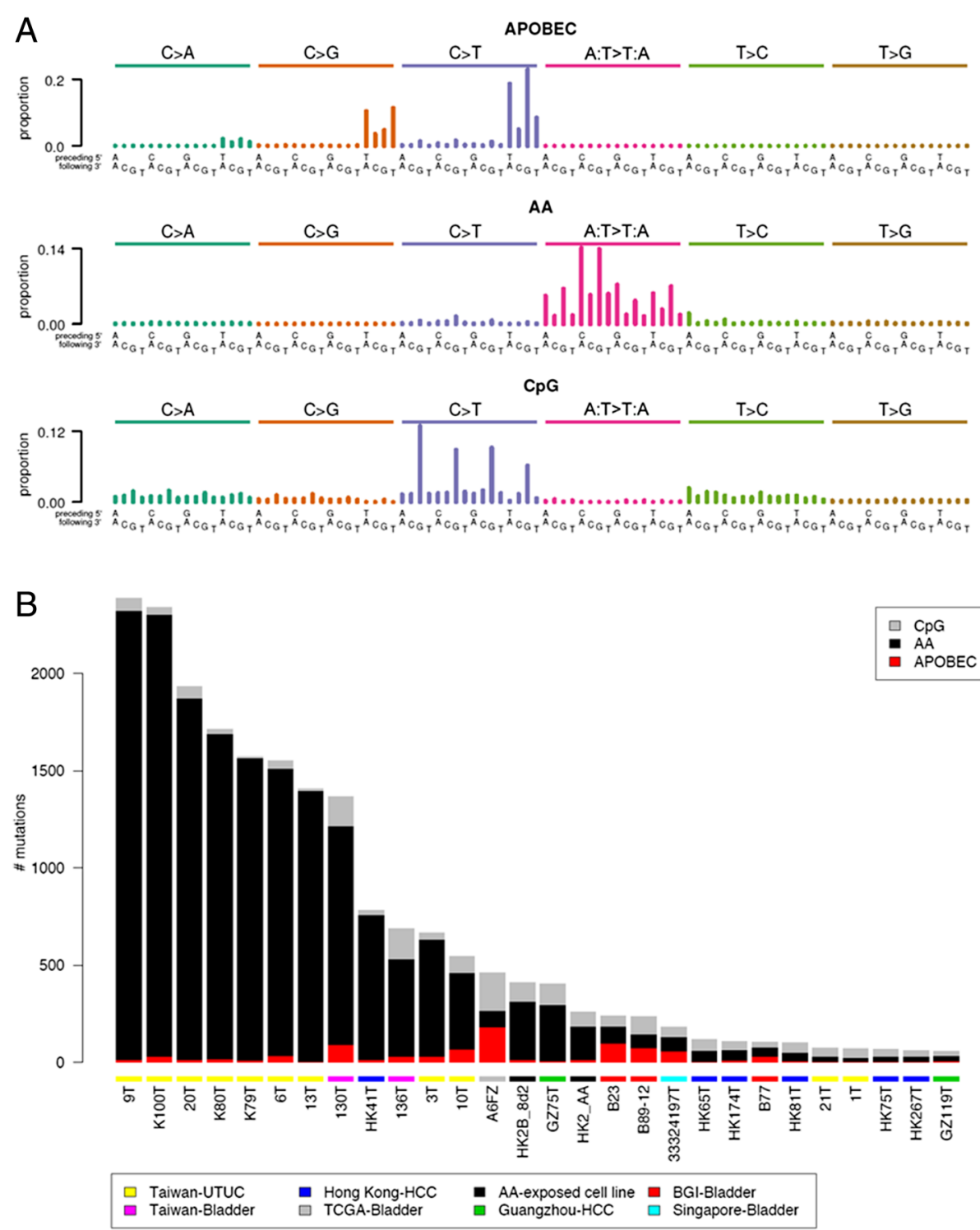

Figure 3 Inferred mutation signatures and their contributions to somatic mutations in bladder cancers, UTUCs, and HCCs. (A) EMu discerned three mutation signatures. The height of each bar (the $y$ axis) represents the proportion of mutations in the inferred signature that fall into a particular trinucleotide mutation class, adjusted for the frequency of the trinucleotide in the exome. (B) The contributions of each mutation signature (that is, inferred mutational process) to the somatic mutations in each tumor in Table 1. BGI-Bladder: tumors reported in reference 36.

Signature 5. We calculated FDRs across the $P$-values of all tumors, adopting a threshold of 0.05 as indicating possible AA exposure. Seven bladder cancers (including the two from patients in Taiwan) met this threshold, with the highest FDR being 0.006 (Table 1). In addition to the two tumors from Taiwan, the bladder cancers with possible AA signatures comprised one out of the 11 tumors treated in Singapore (Figure 2A; Figures S1 and S2 in Additional file 1), three of the 99 tumors treated in China (Figure 2B, Additional file 1: Figures S1, S3) and one of the 194 tumors treated in North America (Figure 2C; Figures S1 and S4 in Additional file 1; Table 1). The mutation spectra of these seven tumors, together with AA-exposed UTUCs and HCCs for comparison, are shown in Figures $1 \mathrm{~A}$ and 2A-C and Figures S1, S5 and S6 in Additional file 1. These tumors included three muscle-invasive tumors (two from Taiwan and one from the United States) and four non-muscle-invasive tumors (one from Singapore and three from China).

We then confirmed this analysis using the second characteristic of AA mutagenesis: bias against $\mathrm{A}>\mathrm{T}$ mutations on the transcribed strand. We calculated $P$-values based on the observed proportions of $\mathrm{A}>\mathrm{T}$ 
mutations on the transcribed and non-transcribed strands and the null hypothesis of an equal number of $\mathrm{A}>\mathrm{T}$ mutations on both strands. All seven bladder tumors had raw $P$-values $<0.0004$ and FDRs $<0.017$ (one-sided binomial tests; Table 1).

All but one of the bladder cancers that were identified as due to, or as possibly due to, AA exposure, based on the criteria above, had patterns of trinucleotide contexts for A:T > T:A mutations that were very similar to the EMu-extracted AA signature (Figure $3 \mathrm{~A}$ ). This was indicated by A:T > T:A cosine similarities $>0.92$ for all the bladder cancers in Table 1 except one. The exception was a tumor of a white, 86-year-old patient who was treated in the United States (TCGA-K4-A6FZ-01A-11DA31L-08). This tumor showed relatively higher rates of $5^{\prime}-\mathrm{TC}-3^{\prime}$ to $5^{\prime}-\mathrm{AC}-3^{\prime}$ (equivalently, $5^{\prime}-\mathrm{GA}-3^{\prime}$ to $5^{\prime}-$ GT-3') mutations (Figure 1C). This was a pattern not seen in the EMu-extracted AA signature (Figure 3A) or in tumors with predominant AA mutations (Figure 1). This dissimilarity was reflected by a cosine similarity of only 0.53 between this tumor and the EMu-extracted A:T > T:A AA signature. Clinical history that would indicate whether this patient used herbal remedies was unavailable. However, this patient was a lifelong nonsmoker, suggesting that tobacco smoke would not have been a source of the $\mathrm{A}>\mathrm{T}$ mutations in this tumor. Thus, the statistically significant over-representation of $\mathrm{A}: \mathrm{T}>\mathrm{T}: \mathrm{A}$ mutations and the strand bias in this tumor may be due not to AA but to another, unknown mutagen.

\section{Discussion}

We have presented evidence of AA mutagenesis in the genomes of bladder cancers in patients known to have been exposed to AA, as well as evidence of AA exposure in a subset of bladder cancers from three patient populations. First, we found evidence of AA exposure in 3 of 99 bladder cancers treated in China. This is consistent with the fact that plants containing AA are components of traditional Chinese medicine [46]. Indeed, AAcontaining herbal remedies are still readily available in China [47]. We also observed one likely AA-exposed case of bladder cancer from Singapore where traditional Chinese medicine is also widely used. Because the patient was 80 years old, he may have been exposed to AA before its use was prohibited in Singapore. Although HCCs were not the focus of this study, consistent with our previous report [14], the analysis here showed that many $\mathrm{HCCs}$ have A:T > T:A mutation patterns very similar to that of the AA signature (Figure 3A). HK41T had a cosine similarity with the EMu-extracted A:T > T:A signature of 0.99 (Figure 1D, Table 1) and GZ75T had a similarity of 0.975 (Table 1).

Our data demonstrating a molecular link between AA and bladder cancer are consistent with previous work showing the presence of AA adducts in the bladders of AA-exposed mice [34,35], with the role of AA in UTUCs $[14,23,24]$, and with previously reported elevated risk of bladder cancer in patients with AA-induced kidney failure [31-33]. However, to our knowledge, the present research is the first to connect AA mutagenesis with bladder cancer in patients from the general population without a history of AA-induced kidney failure.

Although one bladder tumor from Singapore and three bladder tumors from China showed patterns of A:T > T:A mutations that were very similar to those in tumors with known AA exposure (Table 1; Figure S1 in Additional file 1), it remains a formal possibility that these mutations arose from exposure to an unknown mutagen with effects very similar to those of AA. At present, no such mutagen is known, but the mutation signatures of most mutagens remain unstudied. Nevertheless, the reasonable prior possibility of AA exposure through herbal remedies in the tumors from Singapore and China would argue that the mutagen in these tumors was in fact AA.

Associations between exposures and disease are often difficult to establish based on epidemiological data alone $[48,49]$. Various 'omics' approaches, such as transcriptomics, metabolomics, proteomics, and genomics, are now being evaluated as tools for assessing environmental exposures and cancer risk [50]. Here, we provide an example of using recent advances in sequencing technologies to detect a new likely link between AA mutagenesis and bladder cancers, in addition to its links with UTUC, HCC, and RCC. This was possible because the AA signature has been established in cell culture $[14,25]$ and because previous epidemiological data have linked AA to UTUCs $[24,28,51]$.

The medicinal use of AA-containing plants has a long and geographically diverse history and likely remains widespread despite efforts at regulation in some countries [51-54]. This is probably especially true in highly populous countries in Asia: China and India alone have a combined population of $>2.6$ billion, and although use of AA-containing plants in Chinese herbal medicine has received much attention, AA-containing plants are probably also widely used in the Indian subcontinent [53,54]. In addition, there is evidence that AA-containing plants are also used in Central and South America [53]. Although use of these plants does not appear to be widespread in the US, their sale or use as botanical products without claims of health benefits are not restricted there. Under the Dietary Supplement Health and Education Act of 1994, the US Food and Drug Administration has little ability to regulate botanical products, although it has urged manufacturers and distributors to ensure that botanical products are free of AA and advised consumers to not use products that might contain AA [55-57]. 


\section{Conclusions}

Large populations are using traditional herbal medicines that prescribe AA-containing plants, and there is new, rapidly emerging molecular epidemiological evidence of AA exposure in several types of cancer, including bladder cancer, not previously linked to AA. There is also additional exposure to AA due to unintended contamination of food with AA-containing plants. Thus, multiple lines of evidence point to AA exposure as a worldwide public health issue that should be addressed by further investigation and by primary prevention through regulation and public education. In addition to opportunities for primary prevention, knowledge of AA exposure would provide opportunities for secondary prevention in the form of intensified screening of patients with known or suspected AA exposure.

\section{Additional file}

Additional file 1: Supplementary material. Contains Supplementary

Materials and Methods, Supplementary Figures S1 through S10,

Supplementary Tables S1 through S3, and Supplementary References.

\section{Abbreviations}

AA: aristolochic acid; FDR: false discovery rate; HCC: hepatocellular carcinoma; NMF: non-negative matrix factorization; RCC: renal cell carcinoma; TCGA: The Cancer Genome Atlas; UTUC: upper urinary tract urothelial cell carcinoma.

\section{Competing interests}

The authors declare that they have no competing interests.

\section{Authors' contributions}

SLP, MNH, S-TP, PT, BTT, and SGR conceived the study. PT, BTT, and SGR directed the study. LN, W-HW, C-KC, JY, and S-TP were involved in the specimen collection, pathological reviews, and clinical data collections. $\mathrm{MNH}, \mathrm{CY}$, JRM, WY and SGR performed the bioinformatics data analysis. SLP, HLH, AG, SSM, EYS, and LDL performed sequencing and data analysis. SLP, MNH, S-TP, PT, BTT and SGR wrote the manuscript, with the assistance and final approval of all authors. All authors read and approved the final manuscript.

\footnotetext{
Acknowledgments

Supported in part by the Duke-NUS Signature Research Programs, funded by the Singapore Ministry of Health. Supported in part by funding from the Singapore National Medical Research Council (NMRC/STAR/0006/2009), the Singapore Millennium Foundation, the Lee Foundation, the National Cancer Centre Research Fund, The Verdant Foundation, the Duke-NUS Graduate Medical School, the Cancer Science Institute Singapore, the Chang Gung Memorial Hospital (CMRPG370471-3), the Taiwan National Science Council (98-2314-B-182A-057-MY3). WY and LDL are the recipients of the NUS Graduate School for Integrative Sciences and Engineering Scholarship, Singapore. We thank the anonymous reviewers for their comments and suggestions, which distinctly improved the paper. The results published here are in part based upon data generated by TCGA Research Network [37]. These data were generated from samples contributed by Asterand, Baylor College, CHI-Penrose Colorado, Christiana Healthcare, Cleveland Clinic, Cornell Medical College, Gundersen Lutheran Health System, Hartford Hospital, ILSBio, Indiana University Purdue University Indianapolis, Indivumed, International Genomics, Consortium, Lahey Clinic, MD Anderson, Medical College of Georgia, Memorial Sloan Kettering, Ontario Institute for Cancer Research, Roswell Park, University of California San Francisco, University Of Chicago, University of Colorado Denver, University of Miami, University of Minnesota, University of North Carolina, University of Oklahoma HSC, University of Pittsburgh, UT Southwestern Medical Center at Dallas.
}

\section{Author details}

${ }^{1}$ Laboratory of Cancer Epigenome, Division of Medical Sciences, National Cancer Centre Singapore, 11 Hospital Drive, Singapore 169610, Singapore. ${ }^{2}$ Program in Cancer and Stem Cell Biology, Duke-NUS Graduate Medical School, 8 College Road, Singapore 169857, Singapore. ${ }^{3}$ Centre for Computational Biology, Duke-NUS Graduate Medical School, 8 College Road, Singapore 169857, Singapore. ${ }^{4}$ National University of Singapore, Graduate School for Integrative Sciences and Engineering, 28 Medical Drive, Singapore 117456, Singapore. ${ }^{5}$ Department of Urology, Singapore General Hospital, Outram Road, Singapore 169608, Singapore. ${ }^{6}$ Department of Chemical Engineering and Biotechnology, Graduate Institute of Biotechnology, National Taipei University of Technology, 1, Section 3, Chung-hsiao East Road, Taipei 10608, Taiwan. ${ }^{7}$ Division of Urology, Department of Surgery, Chang Gung Memorial Hospital, Linkou, School of Medicine, Chang Gung University, 5, Fusing Street, Gueishan Township, Taoyuan County 333, Taiwan. ${ }^{8}$ Division of Cellular and Molecular Research, National Cancer Centre Singapore, 11 Hospital Drive, Singapore 169610, Singapore. ${ }^{9}$ Cancer Science Institute of Singapore, National University of Singapore, Centre for Life Sciences, 28 Medical Drive, Singapore 117456, Singapore. ${ }^{10}$ Genome Institute of Singapore, 60 Biopolis Street Genome, Singapore 138672, Singapore.

Received: 18 December 2014 Accepted: 2 April 2015

Published online: 28 April 2015

\section{References}

1. Jemal A, Bray F, Center MM, Ferlay J, Ward E, Forman D. Global cancer statistics. CA Cancer J Clin. 2011;61:69-90.

2. Brennan P, Bogillot $O$, Cordier S, Greiser E, Schill W, Vineis P, et al. Cigarette smoking and bladder cancer in men: A pooled analysis of 11 case-control studies. Int J Cancer. 2000;86:289-94.

3. Howe G, Burch J, Miller A, Cook G, Esteve J, Morrison B, et al. Tobacco use, occupation, coffee, various nutrients, and bladder cancer. J Natl Cancer Inst. 1980;64:701-13.

4. Bates MN, Smith AH, Hopenhayn-Rich C. Arsenic ingestion and internal cancers: a review. Am J Epidemiol. 1992;135:462-76.

5. Hopenhayn-Rich C, Biggs ML, Fuchs A, Bergoglio R, Tello EE, Nicolli H, et al. Bladder cancer mortality associated with arsenic in drinking water in Argentina. Epidemiology. 1996;7:117-24.

6. Ward E, Carpenter A, Markowitz S, Roberts D, Halperin W. Excess number of bladder cancers in workers exposed to ortho-toluidine and aniline. J Natl Cancer Inst. 1991;83:501-6.

7. Ward EM, Sabbioni G, DeBord DG, Teass AW, Brown KK, Talaska GG, et al. Monitoring of aromatic amine exposures in workers at a chemical plant with a known bladder cancer excess. J Natl Cancer Inst. 1996;88:1046-52.

8. Baan R, Straif K, Grosse Y, Secretan B, El Ghissassi F, Bouvard V, et al. Carcinogenicity of some aromatic amines, organic dyes, and related exposures. Lancet Oncol. 2008;9:322-3.

9. International Agency for Research on Cancer. A Review of Human Carcinogens: Chemical Agents and Related Occupations. 2012. http:// monographs.iarc.fr/ENG/Monographs/vol100F/mono100F.pdf.

10. Villanueva CM, Fernandez F, Malats N, Grimalt JO, Kogevinas M. Metaanalysis of studies on individual consumption of chlorinated drinking water and bladder cancer. J Epidemiol Community Health. 2003;57:166-73.

11. Poon SL, McPherson JR, Tan P, Teh BT, Rozen SG. Mutation signatures of carcinogen exposure: genome-wide detection and new opportunities for cancer prevention. Genome Med. 2014;6:24.

12. Helleday T, Eshtad S, Nik-Zainal S. Mechanisms underlying mutational signatures in human cancers. Nat Rev Genet. 2014;15:585-98.

13. Alexandrov LB, Stratton MR. Mutational signatures: the patterns of somatic mutations hidden in cancer genomes. Curr Opin Genet Dev. 2014;24:52-60.

14. Poon SL, Pang ST, McPherson JR, Yu W, Huang KK, Guan P, et al. Genomewide mutational signatures of aristolochic acid and its application as a screening tool. Sci Transl Med. 2013;5:197ra101.

15. Nik-Zainal S, Alexandrov LB, Wedge DC, Van Loo P, Greenman CD, Raine K, et al. Mutational processes molding the genomes of 21 breast cancers. Cell. 2012;149:979-93.

16. Alexandrov LB, Nik-Zainal S, Wedge DC, Aparicio SA, Behjati S, Biankin AV, et al. Signatures of mutational processes in human cancer. Nature. 2013;500:415-21 
17. Lawrence MS, Stojanov P, Polak P, Kryukov GV, Cibulskis K, Sivachenko A, et al. Mutational heterogeneity in cancer and the search for new cancerassociated genes. Nature. 2013;499:214-8.

18. Alexandrov LB, Nik-Zainal S, Wedge DC, Campbell PJ, Stratton MR. Deciphering signatures of mutational processes operative in human cancer. Cell Rep. 2013;3:246-59.

19. Fischer A, Illingworth CJ, Campbell PJ, Mustonen V. EMu: probabilistic inference of mutational processes and their localization in the cancer genome. Genome Biol. 2013;14:R39. http://www.sanger.ac.uk/resources/ software/emu.

20. Nordentoft I, Lamy P, Birkenkamp-Demtroder K, Shumansky K, Vang S, Hornshoj $\mathrm{H}$, et al. Mutational context and diverse clonal development in early and late bladder cancer. Cell Rep. 2014;7:1649-63.

21. Burns MB, Temiz NA, Harris RS. Evidence for APOBEC3B mutagenesis in multiple human cancers. Nat Genet. 2013;45:977-83.

22. Roberts SA, Lawrence MS, Klimczak L, Grimm SA, Fargo D, Stojanov P, et al. An APOBEC cytidine deaminase mutagenesis pattern is widespread in human cancers. Nat Genet. 2013;45:970-6.

23. Hoang ML, Chen $\mathrm{CH}$, Sidorenko VS, He J, Dickman KG, Yun BH, et al. Mutational signature of aristolochic acid exposure as revealed by whole-exome sequencing. Sci Transl Med. 2013;5:197ra102.

24. Chen CH, Dickman KG, Moriya M, Zavadil J, Sidorenko VS, Edwards KL, et al. Aristolochic acid-associated urothelial cancer in Taiwan. Proc Natl Acad Sci U S A. 2012;109:8241-6.

25. Olivier M, Weninger A, Ardin M, Huskova H, Castells X, Vallee MP, et al. Modelling mutational landscapes of human cancers in vitro. Sci Rep. 2014:4:4482.

26. Moriya M, Slade N, Brdar B, Medverec Z, Tomic K, Jelakovic B, et al. TP53 Mutational signature for aristolochic acid: an environmental carcinogen. Int J Cancer. 2011;129:1532-6.

27. Hollstein M, Moriya M, Grollman AP, Olivier M. Analysis of TP53 mutation spectra reveals the fingerprint of the potent environmental carcinogen, aristolochic acid. Mutat Res. 2013;753:41-9.

28. Chen $\mathrm{CH}$, Dickman KG, Huang CY, Moriya M, Shun CT, Tai HC, et al. Aristolochic acid-induced upper tract urothelial carcinoma in Taiwan: clinical characteristics and outcomes. Int J Cancer. 2013;133:14-20.

29. Jelaković B, Castells X, Tomić K, Ardin M, Karanović S, Zavadil J. Renal cell carcinomas of chronic kidney disease patients harbor the mutational signature of carcinogenic aristolochic acid. Int J Cancer. 2014. Epub ahead of print.

30. Scelo G, Riazalhosseini Y, Greger L, Letourneau L, Gonzàlez-Porta M, Wozniak MB, et al. Variation in genomic landscape of clear cell renal cell carcinoma across Europe. Nat Commun. 2014;5:5135.

31. Lemy A, Wissing KM, Rorive S, Zlotta A, Roumeguere T, Martinez M-CM, et al. Late onset of bladder urothelial carcinoma after kidney transplantation for end-stage aristolochic acid nephropathy: a case series with 15-year follow-up. Am J Kidney Dis. 2008;51:471-7.

32. Li WH, Yang L, Su T, Song Y, Li XM. Influence of taking aristolochic acidcontaining Chinese drugs on occurrence of urinary transitional cell cancer in uremic uremic patients undergoing dialysis. Zhonghua yi xue za zhi. 2005:85:2487-91.

33. Li XB, Xing NZ, Wang Y, Hu XP, Yin H, Zhang XD. Transitional cell carcinoma in renal transplant recipients: a single center experience. Int J Urol. 2008;15:53-7.

34. Shibutani S, Dong H, Suzuki N, Ueda S, Miller F, Grollman AP. Selective toxicity of aristolochic acids I and II. Drug Metab Dispos. 2007;35:1217-22.

35. Schmeiser HH, Schoepe KB, Wiessler M. DNA adduct formation of aristolochic acid I and II in vitro and in vivo. Carcinogenesis. 1988;9:297-303.

36. Guo G, Sun X, Chen C, Wu S, Huang P, Li Z, et al. Whole-genome and whole-exome sequencing of bladder cancer identifies frequent alterations in genes involved in sister chromatid cohesion and segregation. Nat Genet. 2013:45:1459-63.

37. The Cancer Genome Atlas. http://cancergenome.nih.gov/.

38. The Cancer Genome Atlas Data Portal. https://tcga-data.nci.nih.gov/tcga/

39. Cancer Genome Atlas Research Network. Comprehensive molecular characterization of urothelial bladder carcinoma. Nature. 2014;507:315-22.

40. Sung WK, Zheng H, Li S, Chen R, Liu X, Li Y, et al. Genome-wide survey of recurrent $\mathrm{HBV}$ integration in hepatocellular carcinoma. Nat Genet. 2012;44:765-9.

41. Burrows-Wheeler Aligner. http://bio-bwa.sourceforge.net/.

42. European Genome-phenome Archive, https://www.ebi.ac.uk/ega/home.
43. EMu: Expectation-Maximisation inference of mutational signatures. http://www.sanger.ac.uk/resources/software/emu.

44. The R Project for Statistical Computing. http://www.r-project.org

45. Isa: Latent Semantic Analysis. http://cran.r-project.org/web/packages/lsa/ index.html.

46. Gokmen MR, Cosyns JP, Arlt VM, Stiborova M, Phillips DH, Schmeiser HH, et al. The epidemiology, diagnosis, and management of aristolochic acid nephropathy: a narrative review. Ann Intern Med. 2013;158:469-77.

47. Chau W, Ross R, Li JY, Yong TY, Klebe S, Barbara JA. Nephropathy associated with use of a Chinese herbal product containing aristolochic acid. Med Aust. 2011;194:367-8.

48. Groopman JD, Cain LG, Kensler TW. Aflatoxin exposure in human populations: measurements and relationship to cancer. Crit Rev Toxicol. 1988;19:113-45.

49. Wogan GN. Aflatoxins as risk factors for hepatocellular carcinoma in humans. Cancer Res. 1992;52:2114s-8s.

50. Wild CP, Scalbert A, Herceg Z. Measuring the exposome: a powerful basis for evaluating environmental exposures and cancer risk. Environ Mol Mutagen. 2013;54:480-99.

51. Debelle FD, Vanherweghem $J$, Nortier JL. Aristolochic acid nephropathy: a worldwide problem. Kidney Int. 2008;74:158-69.

52. Grollman AP. Aristolochic acid nephropathy: Harbinger of a global iatrogenic disease. Environ Mol Mutagen. 2013;54:1-7.

53. Heinrich M, Chan J, Wanke S, Neinhuis C, Simmonds MS. Local uses of Aristolochia species and content of nephrotoxic aristolochic acid 1 and 2-a global assessment based on bibliographic sources. J Ethnopharmacol. 2009;125:108-44

54. Michl J, Jennings HM, Kite GC, Ingrouille MJ, Simmonds MSJ, Heinrich M. Is aristolochic acid nephropathy a widespread problem in developing countries? A case study of Aristolochia indica L. in Bangladesh using an ethnobotanical-phytochemical approach. J Ethnopharmacol. 2013;149:235-44.

55. NTP (National Toxicology Program). Report on Carcinogens. 13th ed. Research Triangle Park, NC: U.S: Department of Health and Human Services, Public Health Service; 2014. http://ntp.niehs.nih.gov/pubhealth/roc/roc13/.

56. Marcus DM, Grollman AP. The consequences of ineffective regulation of dietary supplements. Arch Intern Med. 2012:172:1035-6.

57. U.S. Food and Drug Administration. Aristolochic Acid: Safety Alert; 2014. http://www.fda.gov/Food/RecallsOutbreaksEmergencies/ SafetyAlertsAdvisories/ucm095272.htm.

\section{Submit your next manuscript to BioMed Central and take full advantage of:}

- Convenient online submission

- Thorough peer review

- No space constraints or color figure charges

- Immediate publication on acceptance

- Inclusion in PubMed, CAS, Scopus and Google Scholar

- Research which is freely available for redistribution

Submit your manuscript at www.biomedcentral.com/submit 